Sonderabdruck aus

SCHWEIZERISCHE ZEITSCHRIFT FÜR HYDROLOGIE BIRKHÃUSER VERLAG BASEL

Vol. XIX 1957 Facs. 1 


\title{
Ergebnisse neuerer Arbeiten über verschiedene Verfahren zur Desinfektion des Wassers
}

\author{
Von Dr. K. WUHRMANN \\ Chef der biologischen Abteilung der EAWAG
}

Es ist das Ziel der nachfolgenden Ausführungen, über die drei Entkeimungsverfahren für Trinkwasser, nämlich

\author{
UV-Bestrahlung, \\ Desinfektion mit Silber, \\ Desinfektion mit Ozon,
}

einige neuere Erkenntnisse hinsichtlich ihrer Grundlagen, Wirkungsweise und damit ihrer Anwendungsbereiche aufzuzeigen. Es handelt sich um die einzigen zur Zeit technisch ausgereiften Verfahren der «künstlichen» Entkeimung von Wasser (im Gegensatz zur «natürlichen» Desinfektion mittels Langsamfiltration), bei denen keine Geruchs- oder Gescbmacksbeeinträchtigung zu befürchten ist. Die eidgenössische Gesetzgebung fordert eindeutig, dass ein Trinkwasser keinerlei Fremdgeruch oder -geschmack aufweisen darf. Die Erfahrung zeigt leider, dass man aber bei der zur Zeit noch immer bevorzugten Anwendung von Chlor dauernd Schwierigkeiten bei der Aufrechterhaltung dieses wichtigen Grundsatzes antrifft, denn die laufende $\mathrm{Ab}$ gabe eines wirklich entkeimten und gleichzeitig chlorfreien Wassers stellt so hohe Anforderungen an die Kontrolle der Chlordosierung, wie sie nur in grösseren Werken gewährleistet werden kann. Das Bedürfnis für andere Entkeimungsverfahren stellt sich deshalb besonders bei mittleren oder kleinen Wasserversorgungen. Spezielle Aufmerksamkeit verdienen dabei jene Werke, die auf einem Wasser von stark schwankender Qualität basieren (oberflächenbeeinflusste Quellen!). Fast immer ist in diesen Fällen ein konstanter Desinfektionserfolg mittels Chlorung nur dann zu erreichen, wenn periodische, starke Geschmacksbeeinträchtigungen wegen Chlorüberdosierungen in Kauf genommen werden. Unsere Darstellung verfolgt den Zweck, die wichtigsten Grundlagen aufzuzeigen, auf denen eine praktische 
Anwendung der drei Verfahren beruht. Ohne auf technische oder wirtschaftliche Fragen einzutreten, soll auch gezeigt werden, dass sie hinsichtlich der Überwachung der Apparaturen und der Kontrolle ihrer Wirksamkeit eher einfacher und narrensicherer sind als die Chlorungsgeräte und deshalb bei örtlich angepasster Anwendung mindestens gleichviel Vertrauen in der Praxis der Wasseraufbereitung verdienen wie die Chlorung.

\section{Desinfektion von Wasser mittels UV-Bestrahlung}

Die bakterizide Wirkung der Himmelsstrahlung, sei es von direktem Sonnenlicht oder nur von der diffusen Strahlung des blauen Himmels, ist schon lange bekannt (vgl. neuere quantitative Messungen bei BuCHBINDER, SOlOWay und PHelps [4] $]^{\mathrm{T}}$ ); SWART-FüCHTBAUER und RIPPEL-

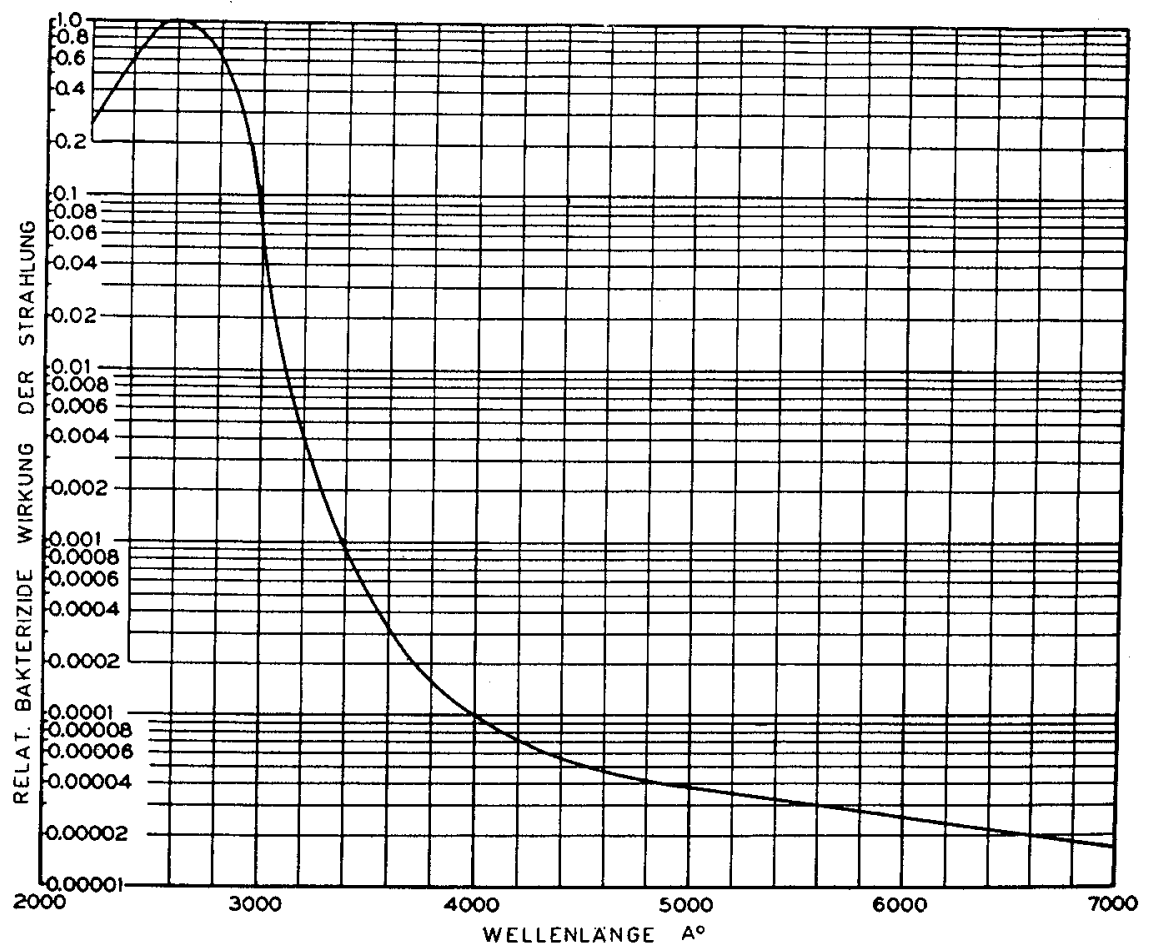

Abbildung I

Relativer Wirkungsgrad von Lichtstrahlung gleicher Energie bei der Abtötung von $E$. coli in Funktion der Wellenlänge. Nach Messungen von Luckiesh, Holladay und TAYLor aus LUCKIESH [17].

1) Die Ziffern in eckigen Klammern verweisen auf das Literaturverzeichnis, Seite 133. 
BALDES [26]). Die wirksamsten Wellenlängen liegen im ultravioletten Gebiet, wobei gewisse Unterschiede in der spektralen Empfindlichkeit einzelner Bakterienarten auftreten (EHRISMANN und NöTHLIN [9]; DUGGARD und HOLLÄNDER [8]). Im wesentlichen erwies sich das Gebiet zwischen 2500 und $2800 \AA$ als das wirksamste. Eine neuere Untersuchung über die Abtötung von Eschericbia coli (LUCKIESH [I7]) in einer Aufschwemmung in Wasser ergab eine spektrale Empfindlichkeitsverteilung für dieses Bakterium gemäss Abbildung I. Unter identischen Versuchsbedingungen fan-

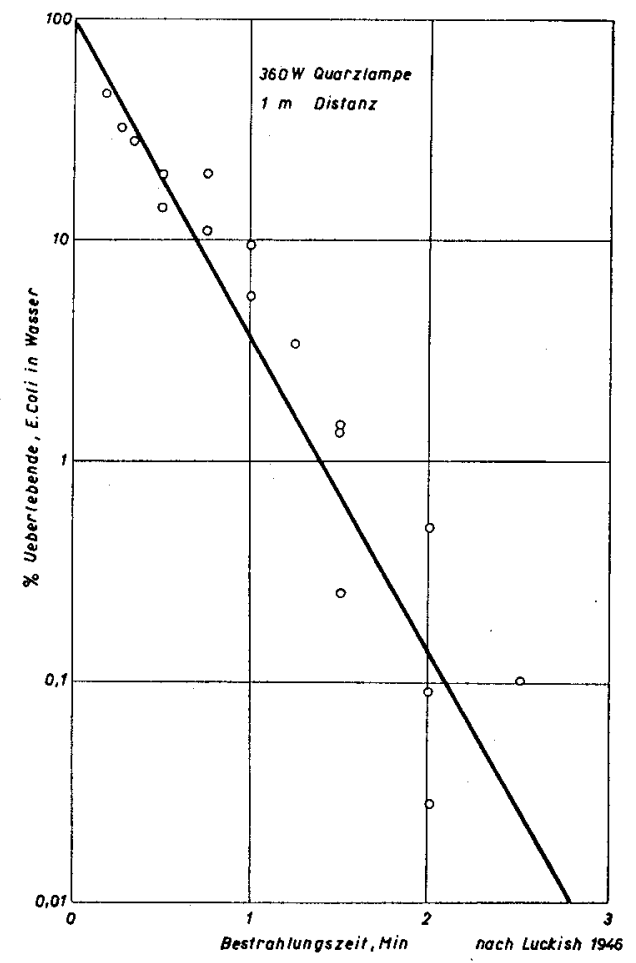

Abbildung 2

Absterbekurve von $E$. coli in dünner Wasserschicht bei Bestrahlung mit ultraviolettem Licht (Quarzlampe $360 \mathrm{~W}, 1 \mathrm{~m}$ Distanz zwischen Brenner und Wasseroberfläche). Nach LUCKIESH [17].

den BuchHolz und Jeney [5] folgende qualitative Abstufung der Abtötungsgeschwindigkeit verschiedener Bakterien $(\lambda=2400-3020 \AA)$ : Salmonella typhosa, S. paratypbi $>$ Shigella dysentheriae, Escherichia coli, Streptokokken $>$ Bacterium prodigiosus, Pseudomonas fluorescens $>$ B. proteus $\gg$ Sporen. 
Die bisher untersuchten Viren scheinen wesentlich weniger schnell inaktiviert zu werden als vegetative Stadien von Bakterien oder sogar Sporen. So fanden DUGGard und HolläNDER [8], dass das TabakmosaikVirus rund zehnmal höhere Energiemengen (bei $\lambda=2650 \AA$ ) als vegetative Keime von B. subtilis und rund achtmal höhere Energiemengen als Subtilissporen zur Abtötung benötigt.

Die Anwendung der UV-Strahlen zur Desinfektion von Trinkwasser wurde auf Grund dieser bakterientötenden Eigenschaft seit langem immer wieder von neuem versucht, ohne dass das Verfahren aber einen breiteren Verwendungskreis gefunden hätte. Es soll nachstehend kurz dargestellt werden, an welche Voraussetzungen die Methode für den Einsatz in der Praxis gebunden ist und welche technischen Lösungen heute in unserem Lande zur Verfügung stehen.

Als wichtigste Voraussetzung für die Dimensionierung einer UV-Desinfektionsanlage muss wie bei allen andern Desinfektionsmitteln die Beziehung zwischen Abtötungseffekt und aufgewendetem, keimtötendem Agens bekannt sein (sogenannte Dosis-Wirkungs-Beziehung). In Abbildung 2 ist eine Kurve für die Abtötung von E. coli in Funktion der Bestrahlungszeit nach LUCKIESH [I7] angegeben.

Diese Funktion kann zugleich auch als Dosis-Wirkungs-Beziehung betrachtet werden, wenn man auf der Abszisse statt der Bestrahlungsdauer die sogenannte Exposition, das heisst das Produkt von Bestrahlungszeit und eingestrahlter Lichtenergie bakterizider Wellenlängen, aufträgt. Auf Grund dieser Dosis-Wirkungs-Kurve kann die zu erwartendeEntkeimungsleistung einer Strahlenquelle berechnet werden, wenn folgende Grössen bekannt sind:

Energieabgabe der Strahlenquelle im wirksamen Wellenlängenbereich $(\lambda=$ rund $2600 \AA)$.

UV-Absorption des Wassers in diesem Wellenlängenbereich.

Einfluss der wichtigsten Aussenfaktoren auf die Abtötungsgeschwindigkeit von Bakterien (zum Beispiel Temperatur und Wasserchemismus).

\section{a) UV-Strabler für die Wasserdesinfektion}

Auf Grund der spektralen Empfindlichkeit der meisten in Frage kommenden Organismen wird eine Strahlungsquelle benötigt, die einen möglichst grossen Anteil der aufgenommenen Energie wieder als Licht im Wellenlängenbereich von $\lambda=$ etwa $2600 \AA$ emittiert. Die Untersuchungen amerikanischer Autoren haben gezeigt, dass dabei zwischen den beiden 
Tabelle 1

Wirkungsgrad von sogenannten $H g$-Hocbdruck- und Niederdruckbrennern binsichtlicb der Emission bakterizider Strablung (LUCKIESH [I7])

\begin{tabular}{|c|c|c|c|c|}
\hline & \multicolumn{2}{|c|}{$\begin{array}{c}\text { Hochdruck } \\
\mathrm{Hg} \text {-Quarzbrenner }\end{array}$} & \multicolumn{2}{|c|}{$\begin{array}{l}\text { Niederdruck } \\
\text { Hg-Brenner }\end{array}$} \\
\hline 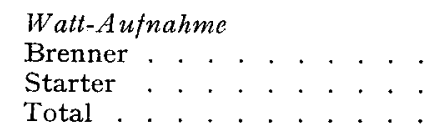 & & $\begin{array}{l}50 \\
50 \\
20\end{array}$ & & $\begin{array}{r}30 \\
9 \\
39\end{array}$ \\
\hline 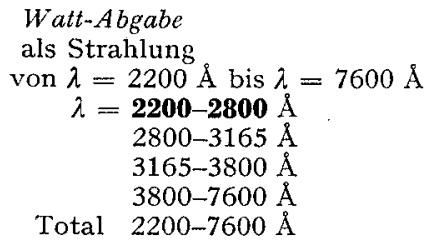 & $\begin{array}{c}\text { Watt } \\
\mathbf{1 0 , 4} \\
10,3 \\
8,3 \\
17,4 \\
46,4\end{array}$ & $\begin{array}{c}\% \text { der } \\
\text { Aufnahme } \\
\mathbf{2 , 4 8} \\
2,45 \\
1,98 \\
4,14 \\
11,05\end{array}$ & $\begin{array}{l}\text { Watt } \\
\mathbf{7 , 9} \\
0,21 \\
0,16 \\
0,9 \\
9,17\end{array}$ & $\begin{array}{c}\% \text { der } \\
\text { Aufnahme } \\
\mathbf{2 0 , 3} \\
0,54 \\
0,41 \\
2,31 \\
23,56\end{array}$ \\
\hline
\end{tabular}

bekannten Arten von Quecksilberbrennern, den sogenannten Hochdruckund Niederdrucklampen, grosse Unterschiede bestehen. In Tabelle I sind die absoluten Energieabgaben im Verhältnis zur Energieaufnahme von zwei Typen von Quecksilberbrennern nach Angaben von LUCKIESH [I7] zusammengestellt, die zeigen, dass für das Spektralgebiet, welches für die Entkeimung von Wasser besonders interessant ist, die Niederdruckbrenner viel mirtschaftlicher arbeiten. Der Unterschied beruht darauf, dass bei den hohen Temperaturen in Hochdruckbrennern die zur Entkeimung wichtigsten Linien des Emissionsspektrums zwischen 2500 und $2600 \AA$ wegen der hohen Konzentration von Quecksilberdampf stark absorbiert werden. Selbstverständlich wirkt die Strahlung eines Hochdruckbrenners trotzdem bakterizid, da ja auch die Emissionslinien der Wellenlängen zwischen 2600 und etwa $3000 \AA$ noch aktiv sind. Aber das Verhältnis zwischen Entkeimungsleistung und Energieaufnahme ist bei ihnen viel ungünstiger als bei den kalten Niederdruckstrahlern.

\section{b) $U V$-Absorption des $W$ assers}

Bei einer gegebenen Strahlungsquelle spielt die Absorption der emittierten Wellenlängen im Gebiete von $2600 \AA$ die wichtigste Rolle für die Abtötungsleistung eines Gerätes. Zunächst muss als selbstverständlich vor- 
Tabelle 2

$U V$-Absorption von Wasser bei $2537 \AA$

\begin{tabular}{|c|c|c|c|}
\hline & $\begin{array}{c}\text { Extinktion } \\
\text { (1 cm) } \\
2537 \AA\end{array}$ & $\mid \begin{array}{c}\% \text { Durchlässigkeit } \\
10 \mathrm{~cm} \text { Schichtdicke } \\
2537 \AA\end{array}$ & $\begin{array}{c}\text { Farbe } \\
\text { (subjektiv) }\end{array}$ \\
\hline 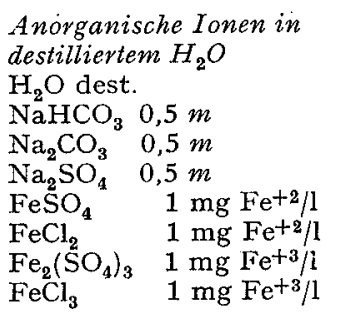 & $\begin{array}{l}\left.0,00091^{1}\right) \\
0,035 \\
0,004 \\
0,019 \\
0,005 \\
0,005 \\
0,031 \\
0,049\end{array}$ & $\begin{array}{l}\left.99,77^{1}\right) \\
44,8 \\
91,4 \\
64,7 \\
89,0 \\
89,0 \\
48,5 \\
32,2\end{array}$ & $\begin{array}{l}\text { klar, farblos } \\
\text { klar, farblos } \\
\text { klar, farblos } \\
\text { klar, farblos } \\
\text { klar, grünlich } \\
\text { klar, grünlich } \\
\text { klar, gelblich } \\
\text { klar, gelblich }\end{array}$ \\
\hline $\begin{array}{l}\text { Natürliche Wässer } \\
\text { Leitungswasser ETH } \\
\text { Grundwasser Tüffenwies } \\
\text { Grundwasser Wädenswil } \\
\text { Quellwasser Lorze } \\
\text { Seewasser Vorfiltrat } \\
\text { Seewasser Langsamfiltrat } \\
\text { Leitungswasser mit } \\
\text { Huminstoffen: a) } \\
\text { b) }\end{array}$ & \begin{tabular}{cc}
0,011 & 0,009 \\
0,006 & 0,006 \\
\multicolumn{2}{c}{0,004} \\
0,004 & 0,005 \\
0,037 & 0,029 \\
0,023 & 0,023 \\
\multicolumn{2}{c}{0,071} \\
0,162
\end{tabular} & 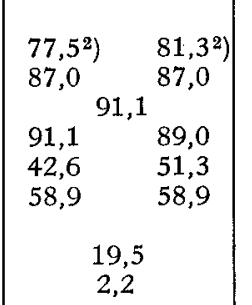 & $\begin{array}{l}\text { klar, farblos } \\
\text { klar, farblos } \\
\text { klar, farblos } \\
\text { klar, farblos } \\
\text { klar, farblos } \\
\text { klar, farblos } \\
\text { schwach gelblich } \\
\text { braun }\end{array}$ \\
\hline
\end{tabular}

ausgesetzt werden, dass das zu behandelnde Wasser durch keinerlei suspendierte Stoffe getrübt ist, da durch sie bereits ein Teil der Strahlen absorbiert wird. Aber auch bei visuell völlig klaren Wässern kommen sehr grosse Unterschiede in der Absorption vor. Einfach destilliertes Wasser besitzt eine sehr grosse UV-Durchlässigkeit; die Absorption beträgt bei $2537 \AA$ nur etwa $0,2 \%$ bei Io $\mathrm{cm}$ Wassertiefe (HaAs [I2]). Entsprechende Angaben einiger Trinkwässer aus der Umgebung von Zürich sind in Tabelle 2 zusammengestellt. $\mathrm{Fe}^{+3}$-Ionen erhöhen die Absorption des Wassers beträchtlich. Eine überraschend starke Absorption weist ferner das Zürichseewasser auf (Fassungstiefe rund $30 \mathrm{~m}$ ), wobei es bemerkenswert ist, dass die absorbierenden Substanzen vermittels der mikrobiologischen Prozesse bei der Langsamfiltration nicht eliminiert werden. Es ist noch unbekannt, um welche Körper es sich in diesem Falle handelt. Immerhin könnte man 
sich vorstellen, dass man es mit Verbindungen aus der Gruppe der Huminsäuren zu tun hat, die bereits in sehr geringen Konzentrationen eine hohe UV-Absorption im fraglichen Wellenlängenbereich aufweisen. Aus diesem Grunde ist auch die UV-Desinfektion von Oberflächenwässern aus moorigen Gebieten, die meist viel Huminsubstanzen aufweisen, in der Regel schwierig. Für die praktische Beurteilung der Belastbarkeit einer UV-Desinfektionsanlage ist deshalb die Kenntnis der Extinktion des Wassers im Gebiete von 2500-2600 $\AA$, die im Laboratorium auf einfache Weise festzustellen ist, unentbehrlich. Die Angaben in Tabelle 2 zeigen, dass wohl für die meisten bei uns zu Trinkzwecken in Frage kommenden Wässer die UV-Behandlung ohne Schwierigkeiten angewendet werden könnte. Auszuschliessen von diesem Verfahren sind aber zum vorneherein jene Wasserherkünfte, die periodisch (zum Beispiel bei Regenfällen) mineralischen Trübungen ausgesetzt sind, da durch die Trübungsstoffe die UV-Absorption sehr stark heraufgesetzt wird und gleichzeitig auch die Gefahr der Bildung von Belägen auf der Oberfläche der Bestrahlungsröhren besteht.

\section{c) Einfluss von äusseren Faktoren auf die bakterizide Wirkung der UV-Strablung}

Leider ist bisher nicht untersucht worden, wie weit die physiologische Wirkung der UV-Strahlung bei Bakterien durch Nebenbedingungen, wie pH und Salzgehalt des Wassers, beeinflusst wird. Man kann mit guten Gründen vermuten, dass diese Faktoren nur einen sehr geringen und praktisch vernachlässigbaren Effekt ausüben. Hinsichtlich der Temperaturwirkung wird von MCCULLOCK [I8] ein Temperaturfaktor von $\Theta_{10}=\mathbf{I}, \mathrm{O}-\mathrm{I}, \mathbf{I}$ angegeben, der praktisch nicht ins Gewicht fällt.

Der Vollständigkeit halber sei an dieser Stelle auch auf die von KELNER [I4], [IS] beschriebene und seither von anderen Autoren bestätigte, sogenannte Photoreaktivierung hingewiesen. KELNER beobachtete bei verschiedenen Pilzsporen, Hefen und $E$. coli, dass nach UV-Bestrahlung $(\lambda=2537 \AA)$ einer Population dieser Zellen ein Teil der Organismen bei sofortiger nachfolgender Bestrahlung mit sichtbarem Licht $(\lambda=5100 \AA)$ reaktiviert wurde und ihre ursprüngliche Vermehrungsfähigkeit wiedergewann. Soweit wir bisher bei Versuchen über die UV-Desinfektion von Wasser feststellen konnten, ist dieser Effekt praktisch allerdings ohne Bedeutung, weil der Prozentsatz photoreaktivierbarer Keime einer Population offenbar ausserordentlich gering ist nach Bestrahlungsintensitäten, wie sie in den Desinfektionsgeräten herrschen. Ferner sind die Voraus- 
setzungen für eine Photoreaktivierung im praktischen Wasserwerksbetrieb normalerweise auch gar nicht vorhanden.

\section{d) Praktisches Ausfübrungsbeispiel}

Auf Grund der Angaben über die Strahlungsleistung von Quecksilberbrennern, die von den betreffenden Fabrikanten oder durch Messung erhalten werden können, und der von LUCKIESH [I7] angegebenen DosisWirkungs-Beziehung, lässt sich diejenige Menge von Wasser mit bekannter Extinktion (bei $\lambda=$ rund $2600 \AA$ ) berechnen, welche pro Zeiteinheit entkeimt werden kann.
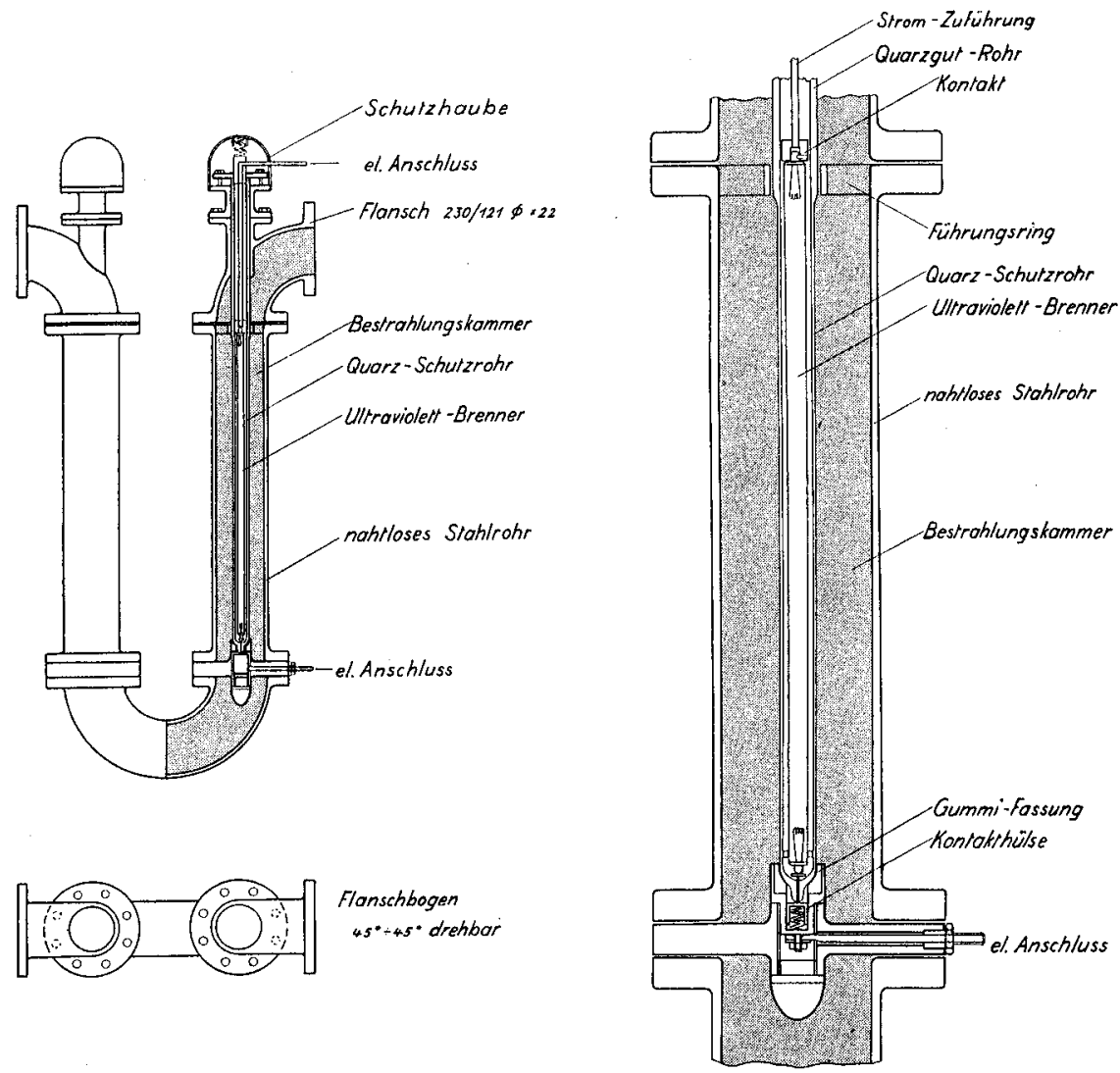

Abbildung 3

Schematische Darstellung und Detail eines UV-Desinfektionsgerätes für grosse Wasserdurchflüsse (vgl. Abb. 4). 
UV-Desinfektionsgerät $k .-s$. mit 2 Bestranlungskammern.

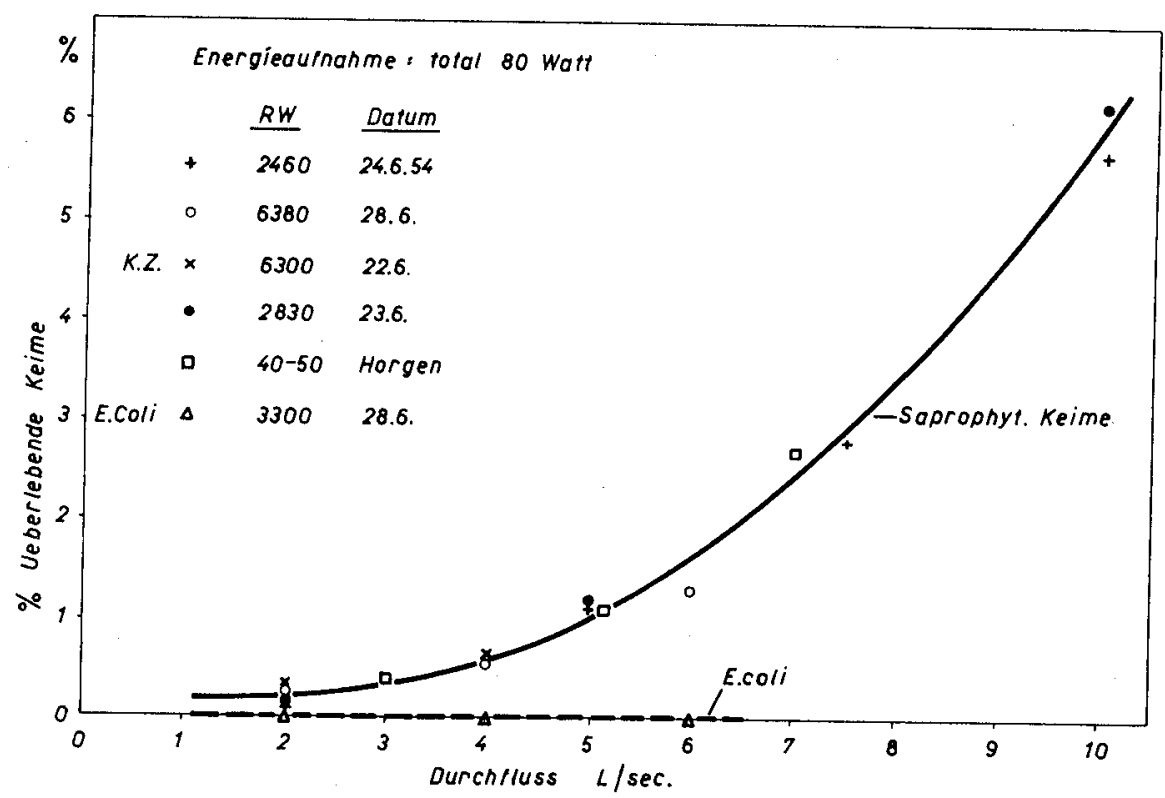

Abbildung 4

Desinfektionseffekt des Gerätes von Abbildung 3 bei verschiedenen Rohwässern (RW) in Abhängigkeit von der Durchflussmenge. Keimzahı des Rohwassers gemäss Kolonne RW.

Technische Lösungen für die laufende Bestrahlung von Wasser sind schon mehrfach vorgeschlagen worden. Naturgemäss beanspruchen jene Konstruktionen das grösste Interesse, die als druckfestes System direkt in ein Verteilungsnetz eingeschaltet werden können. Sie verlangen aber eine technische Durchbildung, die jederzeit das leichte Auswechseln der Quecksilberbrenner gestattet (die Lebensdauer von Niederdruckbrennern beträgt rund 4000-6000 Stunden bei ununterbrochenem Betrieb; bei intermittierendem Betrieb sinkt sie je nach Zahl der Schaltungen) und auch die leichte Reinigung des Brennerschutzrobres ermöglicht. Im übrigen ist es ein hydraulisches Problem, die Wasserströmung in einem Gerät so zu führen, dass die theoretisch verlangte Exposition für jeden mitgeschwemmten Keim erreicht wird. In Abbildung 3 geben wir die Schemazeichnung einer Konstruktion wieder, die wir in ausgedehnten Versuchen überprüfen konnten. Die Apparatur besteht aus zwei hintereinandergeschalteten, zylindrischen Bestrahlungskammern, in welchen axial ein $80 \mathrm{~cm}$ langer 
Niederdruckbrenner in einem Quarzschutzrohr eingesetzt ist. Das Gerät nimmt mit beiden Brennern insgesamt rund $80 \mathrm{~W}$ auf. Seine Entkeimungsleistung bei Zürcher Leitungswasser, welches mit Abwasser infiziert wurde, sowie bei Wasser aus dem Zürichsee (Horgen) und reinen Colisuspensionen ist in Abbildung 4 in Funktion des Wasserdurchflusses angegeben. Die Darstellung zeigt, dass in den verschiedenen Experimenten gut reproduzierbare Desinfektionseffekte erreicht wurden. Die Abtötung von E. coli erfolgte im ganzen geprüften Durchflussbereich in Úbereinstimmung mit der Berechnung zu IOo\%. Saprophytische Keime, unter denen sich im rohen Abwasser viele Sporen befinden, überleben die Behandlung nach Massgabe der Bestrahlungszeit, das heisst der Durchflussgeschwindigkeit im Apparat. Bis zu 4 l/s beträgt für sie die Abtötungsrate über $99 \%$, was für die meisten Fälle genügen dürfte.

Zusammenfassend möchten wir festhalten, dass mit modernen UVStrahlern, die in zweckmässige Bestrahlungskammern eingebaut werden, klare, aber bakteriologisch unzuverlässige Wässer mit einfachen Mitteln entkeimt werden können, wobei die Wartung solcher Anlagen denkbar einfach ist. Die Kontrolle der Geräte ist ohne Schwierigkeit möglich, da einerseits die nützliche Brenndauer der Strahler bekannt und anderseits visuell und mit elektrischen Kontrollschaltungen die Funktion der Brenner leicht zu überwachen ist.

\section{Desinfektion mit Silber}

Obschon der günstige Einfluss von Silber auf verunreinigtes Wasser bereits im Altertum bekannt war und seit den Arbeiten von NäGELI [20] über die Giftwirkung von Silber und anderen Metallen auf lebende Zellen eine riesige Literatur über seine praktische Verwendung und seinen Wirkungsmechanismus bei der Abtötung von Bakterien entstanden ist (vgl. neuere Zusammenfassung bei ZIMMERMANN [32]), hat dieses Desinfektionsverfahren (mindestens in der Schweiz) nicht die Beachtung gefunden, die ihm unseres Erachtens zukommt. Dies beruht zweifellos zum Teil darauf, dass exakte Angaben über die Konzentrations-Wirkungs-Funktion sowie die Beeinflussung dieser Beziehung durch den Wasserchemismus und andere äussere Bedingungen bisher fehlten. Ungenaues Studium der Originalarbeit von NäGELI, zusammen mit dem etwas ungliucklichen Begriff der «Oligodynamie», führten bis in die neueste Zeit wiederholt zu recht merkwürdigen Vorstellungen über die Vorgänge bei der Desinfektion einer Bakteriensuspension durch dieses Metall, weil offenbar auch die 
von SÜPFLE schon I923 veröffentlichte Klarstellung wieder in Vergessenheit geriet. Diese Situation im wissenschaftlichen Schriftum mag nicht wenig zum Misstrauen der Praktiker gegenüber dem Silberverfahren in der Wasseraufbereitungstechnik beigetragen haben. Wir haben an unserem Institut in der letzten Zeit eingehende Untersuchungen über die Entkeimung von Wasser mit Silber durchgeführt, die an anderer Stelle ausführlich veröffentlicht werden sollen (WUHRMANN und ZOBRIST, in Vorbereitung). Im Rahmen dieses Referates möchten wir einige grundsätzliche Erkenntnisse so weit darstellen, als es zur Beurteilung des Verfahrens im Vergleich mit den anderen besprochenen Desinfektionsmethoden notwendig ist.

In der bisherigen Praxis der Wasserdesinfektion mit Silber tritt wohl fast ausschliesslich das Silberion $\mathrm{Ag}^{+}$als bakterizides Agens auf (GAUZE [Io]), sei es, indem metallisches Silber elektrolytisch gelöst wird (zum Beispiel Elektrokatadynverfahren) oder Silber, das an einer Trägersubstanz niedergeschlagen ist, wieder in Lösung gebracht wird (zum Beispiel Hylaverfahren). Schliesslich kann auch durch einfache Dosierung von Silbernitrat der zur Entkeimung notwendige Silbergehalt in einem Wasser erreicht werden. Für die Beurteilung der gegenwärtig zur Verfügung stehenden Anwendungsmethoden von Silber in grösserem technischem Rahmen müssen wir somit die Wirkungsweise von Silberionen und ihre Beeinflussung durch äussere Faktoren kennen.

\section{a) Konzentrations-Wirkungs-Kurve von Silberionen bei E. coli}

Über die Wirkungsweise des Silbers bei der Abtötung von Bakterien weiss man noch sehr wenig. Aus verschiedenen Gründen muss man annehmen, dass die in die Zellen eindringenden Silberteilchen in erster Linie irreversible Verbindungen mit Fermenten eingehen, die zu tödlichen Stoffwechselhemmungen führen. Das gute Komplexbildungsvermögen des Silbers und die zahlreichen, sehr schwer löslichen Verbindungen, die das Metall eingehen kann, lassen für eine solche Wirkungsweise viele Möglichkeiten offen. Da Silberionen, ähnlich wie andere Schwermetallionen, nur sehr langsam in Zellen eindringen, kommt allen Faktoren, welche die Permeabilität des Metalles beeinflussen, eine grosse praktische Bedeutung für den Verlauf derKonzentrations-Wirkungsfunktion bei derKeimabtötung zu. Eine sogenannte bakteriostatische Wirkung des Silbers in pä̈sserigen Lösungen, wie sie von Quecksilberverbindungen bekannt ist (vgl. zum Beispiel ToMCSIK [27]), konnte nicbt nachgewiesen werden. 
Suspendiert man Bakterien in einer Lösung von Silberionen, so tritt wie in anderen Desinfektionsmitteln eine Abtötung der Population ein, deren Verlauf annähernd einer Reaktion erster Ordnung entspricht (vgl. Abb. 5). Die Absterbegeschmindigkeit ist natürlich von der $\mathrm{Ag}^{+}$-Konzentration abhängig, wird aber durch zahlreiche andere Faktoren ebenfalls erheblich beeinflusst.

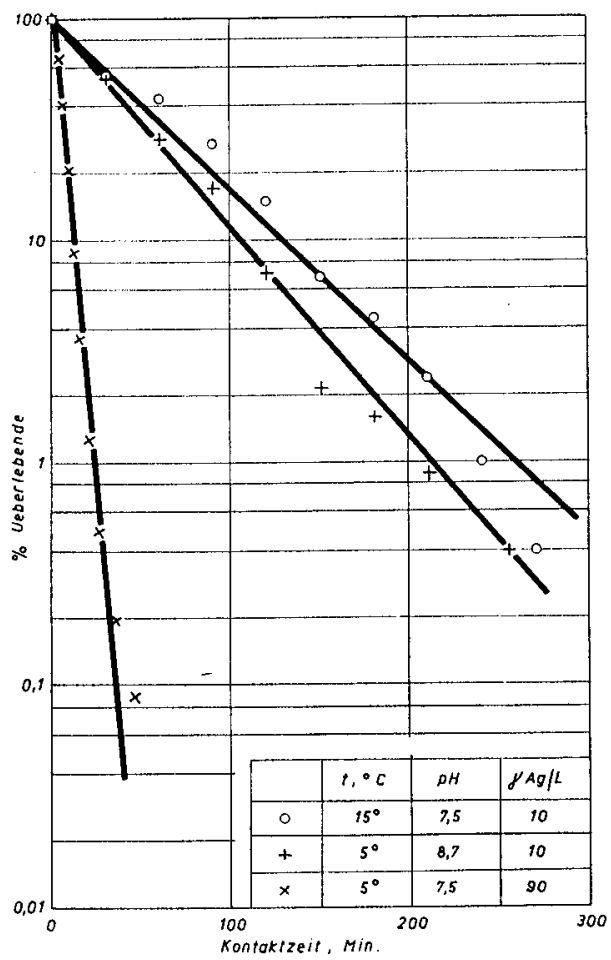

Abbildung 5

Absterbelkurven von $E$. coli in Silberlösungen $\left(\mathrm{AgNO}_{3}\right)$ unter verschiedenen Temperatur- und pH-Bedingungen.

Für durchschnittliche Bedingungen bei der Behandlung eines kontaminierten Oberflächenwassers ergibt sich eine Konzentrations-WirkungsKurve für E. coli gemäss Abbildung 6. Aus der Figur ist die empirisch schon lange bekannte Tatsache ersichtlich, dass die Silberwirkung relativ langsam erfolgt. Nach unserer Vermutung ist dies ausschliesslich der verhältnismässig geringen Permeabilitätsgeschwindigkeit des Silbers zuzu- 
schreiben, so dass viel Zeit verstreicht, bis im Zellinnern die für eine Schädigung notwendige Zahl von Metallmolekeln vorhanden ist. Gemäss unserem Beispiel in Abbildung 6 wird mit einer Silberkonzentration von I00 $\gamma / 1$ eine 99,9prozentige Abtötung von $E$. coli in rund 60 Minuten bei $15^{\circ}$ und $\mathrm{pH} 7,5$ erreicht. Erhöhung bzw. Erniedrigung der Temperatur um

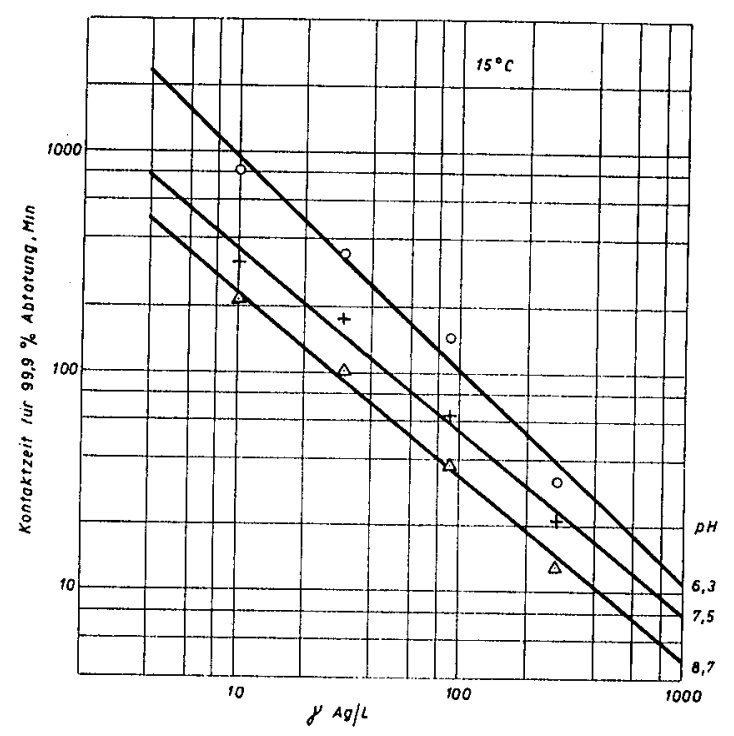

Abbildung 6

Konzentrations-Wirkungs-Kurve von Silber $\left(\mathrm{AgNO}_{3}\right)$ bei der Abtötung von $E$. coli bei $15^{\circ} \mathrm{C}$ und verschiedenen $\mathrm{pH}-$ Werten der Lösung.

$10^{\circ}$ bewirkt eine Verkürzung bzw. Verlängerung dieser Abtötungszeit um das rund $\mathrm{I}, \mathrm{Sfache.} \mathrm{Dieser} \mathrm{Temperaturfaktor} \mathrm{ist} \mathrm{auffallend} \mathrm{gering} \mathrm{(Ozon:}$ $\Theta_{10} \approx 2,3 ;$ Chlor: $\Theta_{10} \approx 2$ ), muss aber bei der Dimensionierung einer Anlage trotzdem berücksichtigt werden. Die Wirkungsgeschwindigkeit von Silberionen wird in bemerkenswertem Umfang durch den $\mathrm{pH}-W$ ert des Wassers beeinflusst. Die Kontaktzeit nimmt bei gegebener Silberkonzentration und Temperatur zwischen $\mathrm{pH} 6,5$ und 8,5 um das rund I, 5fache ab, wenn der pH-Wert um eine Einheit steigt bzw. die Wasserstoffionenkonzentration um eine Zehnerpotenz abnimmt. Die Gründe für diese Erscheinung sind noch durchaus unklar. Rein äusserlich gleicht dieses Verhalten von Silberlösungen der Beeinflussung der Chlorwirkung durch die Wasserstoffionenkonzentration. Bei Chlor sind aber eindeutig die Disso- 
ziationsverhältnisse des Hypochlorits für die Abhängigkeit der Giftwirkung vom $\mathrm{pH}$ verantwortlich zu machen, während in ionalen Silberlösungen dieses Argument wegfällt.

Wir haben sämtliche Kombinationen einer Reihe von Temperatur-, $\mathrm{pH}$ und Silberkonzentrationswerten in ihrer bakteriziden Wirkung geprüft

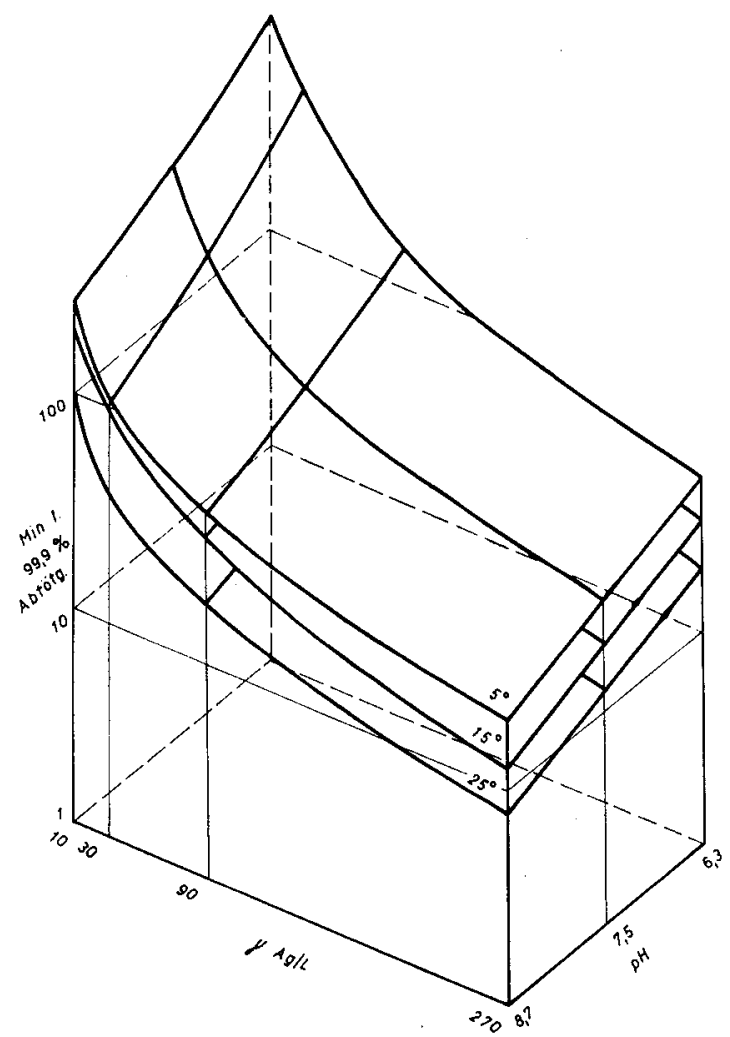

Abbildung 7

Konzentrations-Wirkungs-Beziehung von Silber $\left(\mathrm{AgNO}_{3}\right)$ bei der Abtötung von $\mathrm{E}$. coli bei 3 Temperaturen und 3 pH-Werten der Lösung.

und konnten daraus die räumliche Darstellung von Abbildung 7 ableiten, welche die Abhängigkeit der Absterbezeit von E. coli von diesen Faktoren zusammenfasst. 
b) Abbängigkeit der Silbermirkung vom Wassercbemismus

Im Gegensatz zur UV-Behandlung wird die Desinfektionswirkung von Silber viel weniger von Trübungsstoffen im Wasser beeinträchtigt. Hingegen ist zu beriicksichtigen, dass unter anderem Chlorid- und Sulfidionen sehr schwer lösliche Verbindungen mit Silber eingehen und damit die Konzentration von Silberionen in einer Lösung herabsetzen können. Die theoretisch in chlorid- oder sulfidhaltigen Lösungen bestimmter Konzen-

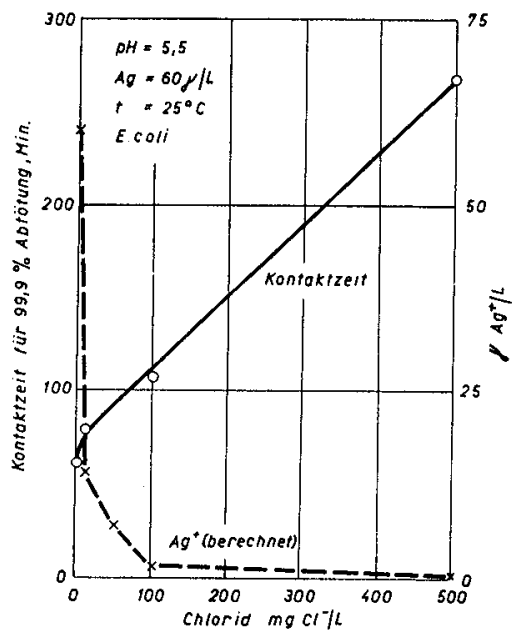

Abbildung 8

Abhängigkeit der Giftwirkung und Silberionenkonzentration einer Silberlösung $\left(\mathrm{AgNO}_{3}\right)$ vom Gehalt an Chloridionen. Die Kurve für die Konzentration von $\mathrm{Ag}^{+}$wurde auf Grund des Löslichkeitsproduktes von $\mathrm{AgCl}$ berechnet.

tration in Lösung bleibenden Silbermengen lassen sich aus den Löslichkeitsprodukten der entsprechenden Silbersalze berechnen. Da Sulfide in einem Trinkwasser normalerweise nicht vorkommen, können wir uns auf die Darstellung des Cblorideinflusses beschränken. Aus Abbildung 8 ergibt sich, dass bei den in unseren Trinkwässern auftretenden Chloridkonzentrationen bis zu maximal etwa $40 \mathrm{mg} \mathrm{Cl}-/ 1$ ein nur unwesentlicher Anstieg der Kontaktzeit für eine bestimmte Silberkonzentration resultiert. Auch bei höheren Chloridkonzentrationen nimmt die Silberwirkung langsamer $\mathrm{ab}$, als es der Konzentrationsabnahme der Silberionen bei zunehmenden Chloridmengen in den Lösungen entspricht. Man muss annehmen, dass die in sehr verdünnten Lösungen entstehenden AgCl-Partikel mindestens teilweise noch in die Zellen permeieren können und das Silber noch zur Wirkung gelangt. 
Kalziumionen üben im untersuchten Bereich zwischen o und 32 französischen Härtegraden nur einen geringen Einfluss auf die Geschwindigkeit der Bakterienabtötung im Sinne einer Verlangsamung mit zunehmender Wasserhärte aus. Organische Verunreinigungsstoffe in Konzentrationen, wie sie in Trinkwässern vorkommen können, sind im Gegensatz zu den oxydativ wirkenden Desinfektionsmitteln, wie Chlor und Ozon, praktisch ohne Einfluss auf die Aktivität einer Silberlösung.

\section{c) Empfindlichkeit einzelner Bakterienarten}

Die grosse Zahl von vergleichenden Untersuchungen über die Empfindlichkeit verschiedener vegetativer Keime zeigt übereinstimmend, dass die pathogenen Arten, welche durch Wasser übertragen werden können, allgemein wesentlich schneller abgetötet werden als nichtpathogene Saprophyten (vgl. ALHA [2]). Die Verhältnisse liegen in dieser Hinsicht also ähnlich wie bei andern Desinfektionsmitteln. Die meisten pathogenen Arten sind auch wesentlich empfindlicher als der übliche Testorganismus E. coli. Unter den Saprophyten bestehen sehr grosse Empfindlichkeitsunterschiede. So wird zum Beispiel Pseudomonas fluoreszens, der in Oberflächenwässern häufig vorkommt, nach unseren Beobachtungen viel schneller abgetötet als $E$. coli, während andere Arten sich als bedeutend widerstandsfähiger erweisen. Dauerformen von Mikroorganismen sind naturgemäss besonders resistent. Immerhin lässt sich die in der tropischen Wassertechnik wichtige Zyste von Entamoeba bistolytica noch ziemlich leicht abtöten (NEwTON und JoNEs [I9]). Dagegen scheinen Bakteriensporen für Silber weitgehend undurchlässig zu sein. Man sollte deshalb bei der Anwendung des Silberverfahrens zum vorneherein auf die Forderung nach einer mehr oder weniger vollständigen Abtötung aller in einem Wasser angetroffenen Keime verzichten, da sonst Kontaktzeiten vorgesehen werden müssen, die ein Vielfaches derjenigen betragen, die zur Desinfektion des Wassers notwendig sind.

\section{d) Praktische Anwendung des Silberverfabrens}

Die Silberdosis, welche einem Wasser zugesetzt wird, behält ihre Wirkung theoretisch über unbegrenzte Zeit bei, wenn einmal die Oberflächen von Leitungen, Behältern oder Filtern mit adsorbiertem Silber gesättigt sind, denn der Silberverbrauch durch die Bakterien ist vernachlässigbar klein. Das Verfahren ist deshalb überall dort zum Einsatz prädestiniert, wo eine gewisse Dauerwirkung des Entkeimungsmittels verlangt wird, 
weil zum Beispiel sekundäre Verunreinigungen des gefassten Wassers möglich sind. Die Anwendung von Silber in geeigneter Form ist deshalb auch bei Badeanlagen gegeben, wobei die algizide Wirkung eine erwünschte Nebenerscheinung darstellt. Die relative Unempfindlichkeit der Desinfektionswirkung gegenüber Schwankungen im Wasserchemismus, und die völlige Geruchs- und Geschmacklosigkeit des behandelten Wassers, unabhängig von der Silberkonzentration, gestalten die Dosierung besonders einfach.

In diesem Zusammenhang sei noch auf die gelegentlichen Behauptungen gesundheitlicher Schädigungen des menschlichen Organismus durch Silber eingetreten, die schon geäussert wurden. Aus den Vereinigten Staaten sind zahlreiche grosse, kommunale Wasserversorgungen bekannt, die ein Trinkwasser mit beträchtlichem natürlicbem Silbergehalt verteilen (vgl. die Zusammenstellung bei BRAIDECH und EMERY [3]), ohne dass je nachteilige Wirkungen festgestellt werden konnten. Es ist auch leicht einzusehen, dass unter den chemischen Bedingungen im menschlichen Verdaungstrakt das oral eingenommene Silber sofort zu Verbindungen umgesetzt wird (Sulfid, Chlorid, organische Komplexe), die nicht resorbierbar sind. Vom biochemischen Gesichtspunkt aus muss also die Wasserdesinfektion mit Silber zweifellos als ganz unbedenklich beurteilt werden.

Auf Grund der heute bekannten Angaben über die KonzentrationsWirkungs-Funktion kann die Dimensionierung einer Entkeimungsanlage und besonders die Festlegung der notwendigen Grösse der Kontaktbehälter zuverlässig erfolgen. Die benötigten Apparaturen sind denkbar einfach, speziell wenn das elektrolytische Lösungsverfahren angewendet wird, so dass auch unter primitiven Verhältnissen eine dauernde Desinfektionsleistung erwartet werden kann. Solche Lösungsapparaturen verlangen bei der Behandlung von Wässern mit hohen Karbonathärten allerdings insofern eine gewisse Wartung, als durch regelmässiges Umpolen der Elektroden eventuelle Kalkabscheidungen an den Kathoden abgelöst werden müssen.

\section{Desinfektion von Wasser mit Ozon}

Als vor einigen Jahren in der Schweiz das Interesse an der Desinfektion von Wasser mittels Ozon erneut erwachte, zeigte es sich, dass über die Chemie und Analytik wässeriger Ozonlösungen überraschend wenig zuverlässige Angaben erhältlich waren, obschon die Anwendung von Ozon zur Wasserentkeimung viel früher als zum Beispiel die Chlorung vorge- 
schlagen und in praktischen Versuchen geprüft worden war (OHLMüLLER [2I]). Der Mangel an einer zuverlässigen analytischen Methode war zweifellos auch für die ausserordentlich widersprechenden, quantitativen Angaben über die bakterizide Wirkung von Ozon verantwortlich (vgl. zum Beispiel Torricelli [28], Buffle [6], Leiguarda et al. [I6], HoLLUTA und UNGER [13]).

Nachdem eine genaue Bestimmungsmethode für Ozon in Wasser in unserem Institut ausgearbeitet worden war (ZEHENDER und STUMM [3 I]), die wir in der Folge als Mangan-ortbo-Tolidin-Methode bezeichnen, konnten zunächst in reinem, ozonzehrungsfreiem Wasser quantitative Angaben über die Giftigkeit von Ozon für Bakterien gewonnen werden (WUHRMANN und MEYRaTH [30]). Kurz zusammengefasst zeigte sich folgendes:

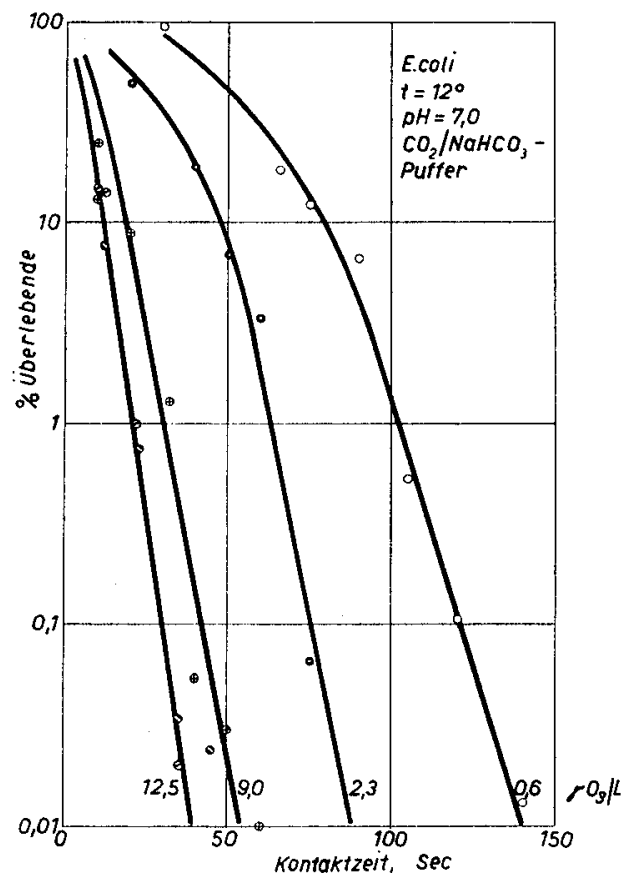

Abbildung 9

Absterbekurven von E. coli in Ozonlösungen verschiedener Konzentration bei $12^{\circ} \mathrm{C}$ und pH 7,0 .

a) Bakterizide Wirkung von ozonisiertem, ozonzebrungsfreiem Wasser

In ozonzehrungsfreiem Wasser mit konstantem Ozongehalt sterben vegetative Keime und Sporen nach ähnlichen Gesetzmässigkeiten ab wie 
in Chlorlösungen, das heisst, die Absterbekurpe ist im logarithmischen Massstabe mindestens in der Anfangsphase gekrümmt (vgl. Abb.9). Die Absterbegeschwindigkeit ist aber wesentlich höher als bei allen andern bisher bekannten Desinfektionsmitteln unter sonst vergleichbaren Bedingungen. Die Konzentrations-Wirkungs-Kurve von Ozon für E. coli in Abbildung Io zeigt, dass zum Beispiel von roooo Bakterien nach zwei Minuten Aufenthaltszeit in einer Lösung mit $0,9 \gamma \mathrm{O}_{3} / 1$ nur noch ein einziger lebender

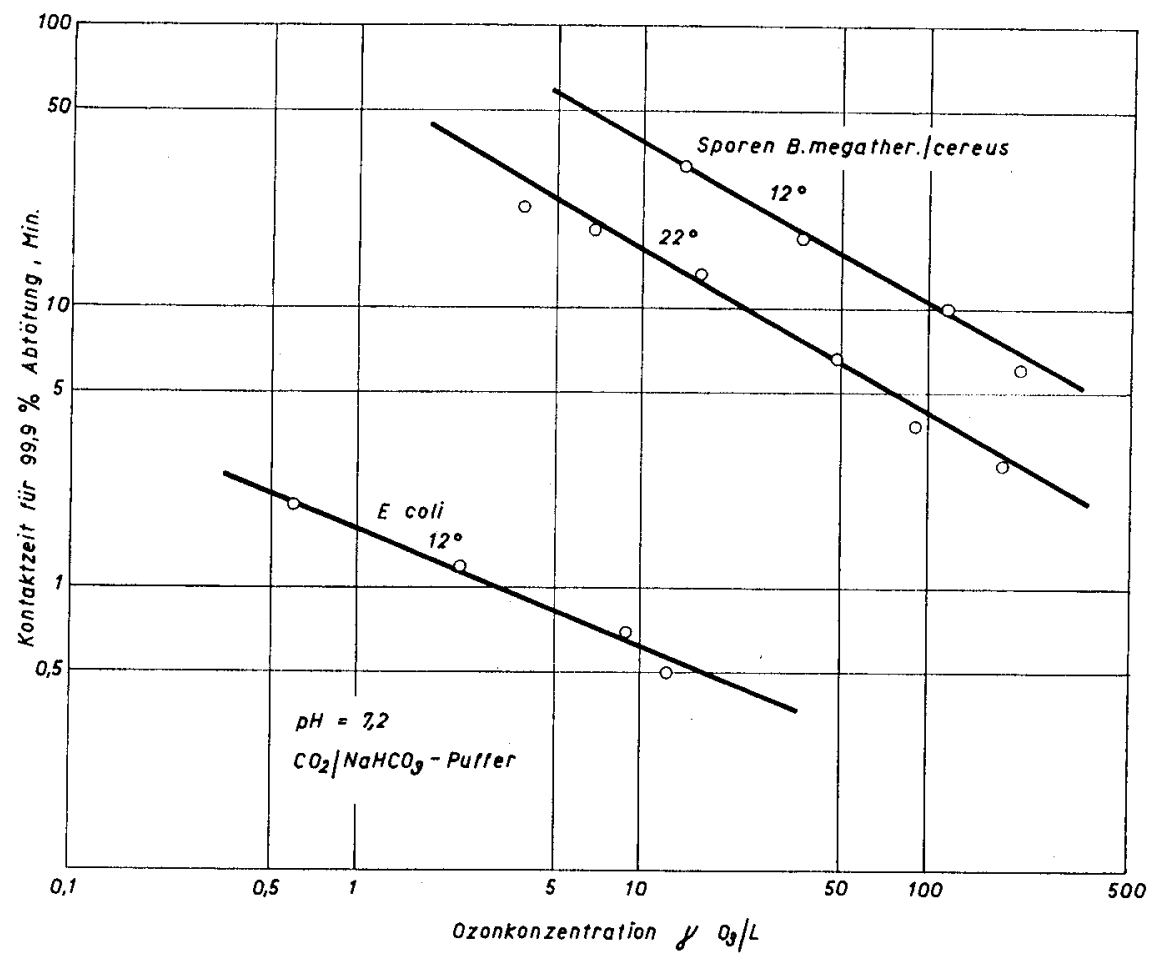

Abbildung 10

Konzentrations-Wirkungs-Kurve von Ozonlösungen für $E$. coli $\left(12^{\circ} \mathrm{C}\right)$ und Sporen eines Bazillus aus der Megatherium-cereus-Gruppe $\left(12^{\circ}\right.$ und $\left.22^{\circ} \mathrm{C}\right)$ bei $\mathrm{pH} 7,2$.

Keim übrigbleibt. Wir können diese überaus hohe Giftigkeit des Ozons heute noch nicht erklären. Sie beruht offenbar zum Teil auf einer sehr guten Permeabilität der Zellhüllen von Bakterien für die giftig wirkenden Partikel, die in einer Ozonlösung vorkommen. Dieser Schluss drängt sich vor allem aus der auffallend raschen Abtötung von Bakteriensporen durch 
Ozon auf. Über den Wirkungsmechanismus und den Angriffsort der in die Zellen eingedrungenen, giftigen Teilchen wissen wir aber noch nichts.

Die hohe Wirkungsgeschwindigkeit von Ozon verwischt naturgemäss die Empfindlichkeitsunterschiede zwischen verschiedenen Keimarten. So werden zum Beispiel E. coli, Staphylococcus albus und Ps. fluorescens unter identischen Bedingungen in annähernd gleichen Zeiten abgetötet. Immerhin finden sich in Mischpopulationen von Saprophyten, wie sie zum Beispiel in Abwässern vorkommen, teilweise recht widerstandsfähige Arten. Die Entkeimungskurve für eine mit Abwasser verunreinigte Lösung besitzt deshalb nicht mehr einen annähernd linearen Verlauf, sondern einen lang ausgezogenen Schwanz, wie man dies auch bei anderen Desinfektionsmitteln unter solchen Bedingungen feststellt. Allerdings sind in Ozonlösungen die Abtötungszeiten auch der widerstandsfähigsten Keime noch wesentlich kürzer als zum Beispiel in Chlorlösungen.

\section{b) Entkeimungswirkung ozonisierter, natürlicher Wässer}

Der natürliche Zerfall von Ozon im Wasser wird durch Hydroxylionen (zunehmender pH-Wert) stark beschleunigt (STUMM [23]). In ähnlicher Weise wirken zahlreiche, in natürlichen Gewässern vorkommende Substanzen, deren Chemismus uns aber grösstenteils noch unbekannt ist. Aus der Tatsache, dass Wasserstoffperoxyd einen Katalysator für den Ozonzerfall darstellt, darf man schliessen, dass Peroxyde, die bei der Ozonisierung aus Verunreinigungsstoffen des Wassers entstehen können, wesentlich zu der Verstärkung des natürlichen Ozonzerfalles beitragen. Bei der Einleitung eines Ozon-Luft-Gemisches in natürliche Wässer ergibt sich deshalb je nach Wasserherkunft eine andere Kurve für den Konzentrationsanstieg gelösten Ozons. Auf Grund der Ozonisierungskurven von vielen natürlichen Wässern, die im Laufe der letzten Jahre in unserem Laboratorium aufgenommen wurden, liess sich eine gewisse Klassifikation der Reaktionsweisen bei der Ozonbegasung vornehmen (STUMM [24]). Im Prinzip können wir vier verschiedene Fälle unterscheiden (Abb. II), die wir vorläufig wie folgt interpretieren:

I. Reinstes, ozonzehrungsfreies Wasser, in welchem gelöstes Ozon nach beginnender Begasung sofort nachzuweisen ist. Die $\mathrm{O}_{3}$-Konzentration steigt gemäss der üblichen Sättigungskurve bis zum theoretischen Höchstwert.

2. Wasser mit ozonzehrenden Stoffen, die nach ihrer Oxydation keinen Einfluss auf die Zerfallsgeschwindigkeit auf Ozon ausüben. Die $\mathrm{O}_{3}$ - 
Konzentration steigt nach einer Initialphase gemäss der theoretischen Sättigungskurve bis zum Sättigungswert.

3. Wasser mit ozonzehrenden Stoffen, die nach ihrer Oxydation als Zerfallsbeschleuniger wirken. Die $\mathrm{O}_{3}$-Konzentration steigt nach der Initialphase langsamer an, als der Theorie entsprechen würde. Die Sättigungskonzentration erreicht nicht den theoretischen Wert.

4. Wasser mit ozonzehrenden Stoffen, die mittels Ozon zu Verbindungen umgewandelt werden, welche den Ozonzerfall dermassen beschleunigen,

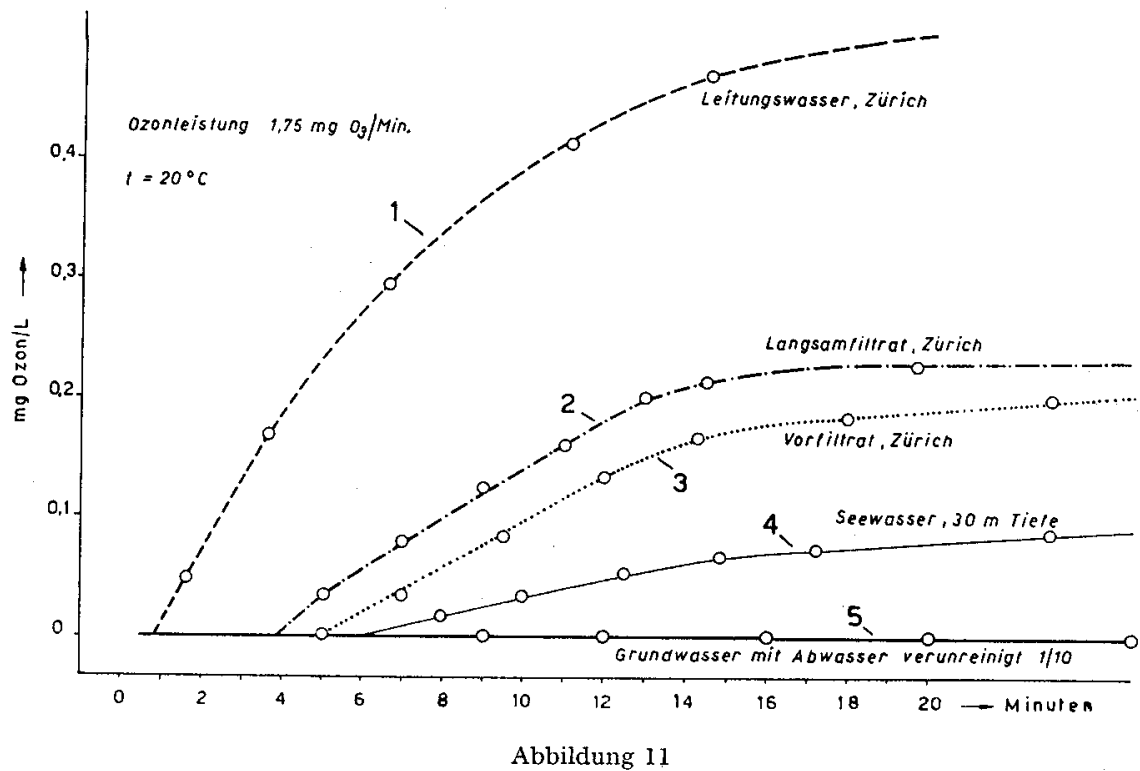

Verlauf des Anstieges der Ozonkonzentration in verschiedenen Wassertypen bei Einleitung eines Ozon-Luft-Gemisches unter identischen Bedingungen. $t=20^{\circ} \mathrm{C}$. Ordinate: gelöstes Ozon (Mangan-o-Tolidin-Methode); Abszisse: Zeit nach Beginn der Gaseinleitung.

dass die Lösungsgeschwindigkeit des Gases kleiner oder höchstens gleich der-Zerfallsgeschwindigkeit wird. Ozon lässt sich mittels der Mangan-ortbo-Tolidin-Methode überhaupt nie nachweisen.

Über den Chemismus der Ozonzerfallsprodukte, die in Wasser entstehen, ist nichts Sicheres bekannt. WeISS [29] bzw. ALDER und HILL [r] geben Verbindungen vom Typus $\mathrm{HO}_{3}{ }^{+}, \mathrm{HO}_{2}, \mathrm{HO}$ und $\mathrm{O}_{2}{ }^{-}$als mögliche intermediäre Substanzen an.

Bei den Wässern des Typus 3 oder 4 kann man beobachten, dass bei völlig negativem Ausfall der Mn-o-Tolidin Reaktion nach Ozonisierung 
dennoch eine Entfärbung von Indigo durch das ozonisierte Wasser erfolgt (Ozonreaktion nach DorTa-SCHAEPPI und TrEadwELl [7]). Ozonisiert man Wasser vom Typus I und 4 so lange, bis beide denselben Oxydationswert, gemessen an der Indigoentfärbung, aufweisen, so zeigen sie an-

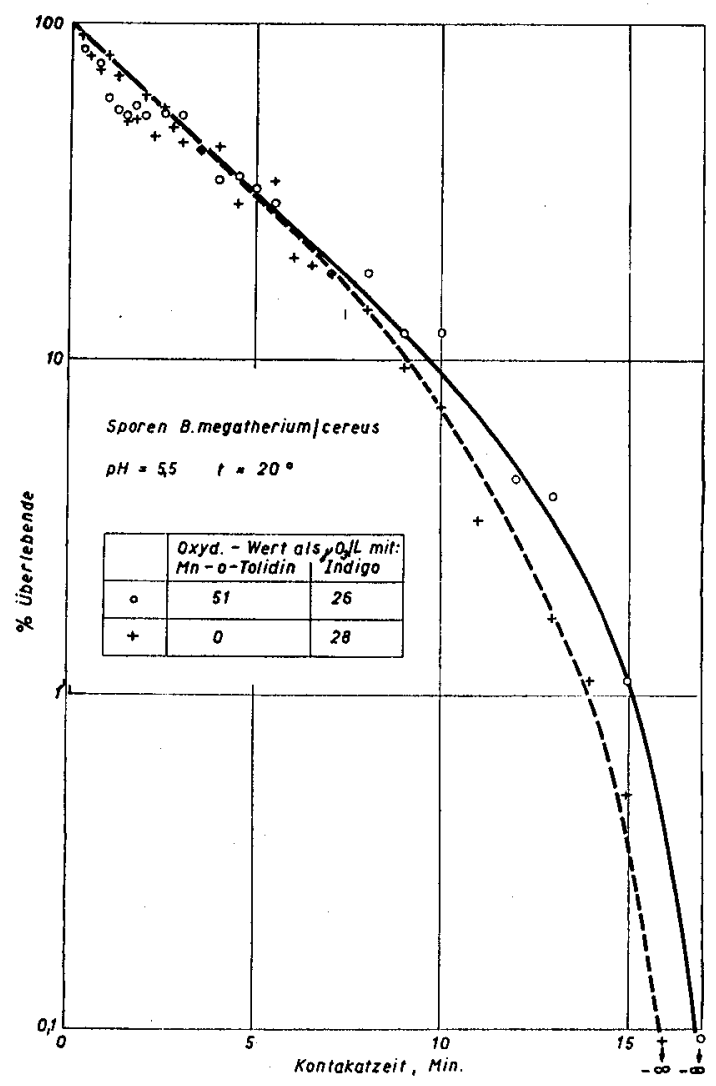

Abbildung 12

Absterbekurven von Sporen eines Bazillus aus der Gruppe megatherium-cereus in ozonisierten Wässern. $O=$ Wasser vom Typus 1 , keine Ozonzehrung nachweisbar. $+=$ ozonisiertes Wasser vom Typus 4, mit Mn-o-Tolidin keine Reaktion, mit Indigo annähernd gleiche Reaktion wie Lösung $\bigcirc$.

nähernd identische bakterizide Wirkung (Abb. I2), obschon eine gleichzeitige Reaktion mit Mn-o-Tolidin nur im Wasser vom Typus I zu beobachten ist. Die Giftigkeit der ozonisierten Wässer scheint demnach in erster Linie durch den zum Beispiel mittels Indigo messbaren Oxydationswert 
bestimmt zu sein, während die Intensität der Mn-o-Tolidin-Reaktion in Wässern mit Ozonzehrung nicht unbedingt dafür massgeblich ist. Als weiteres Beispiel für diese Tatsache sei Abbildung I3 wiedergegeben. Sie zeigt die Absterbekurve von E. coli in ozonisiertem Wasser, welches eine

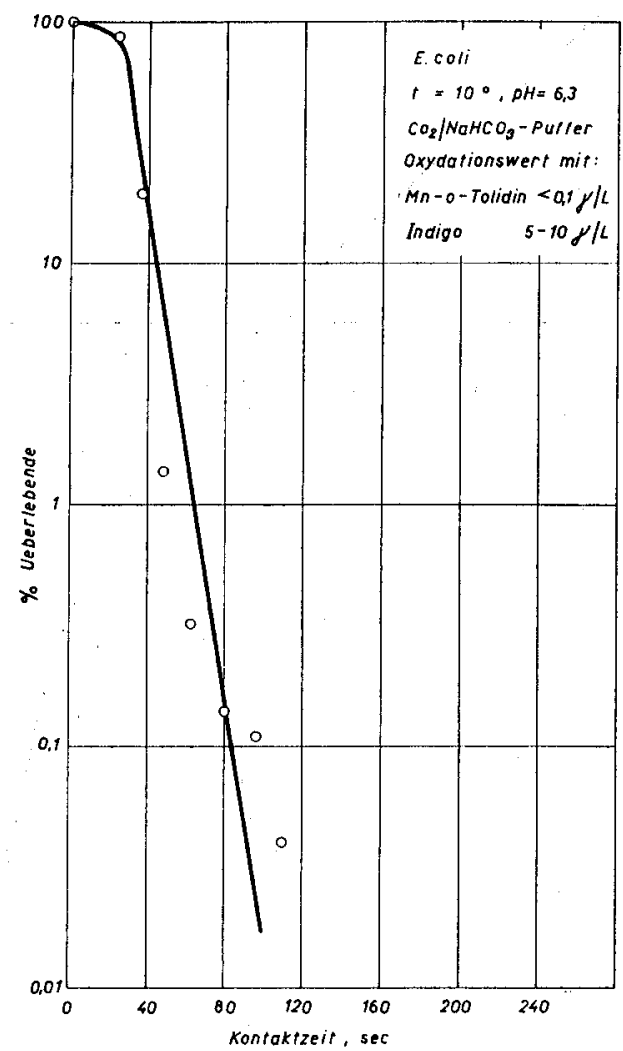

Abbildung 13

Absterbekurve von $E$. coli in ozonisiertem Wasser vom Typus 4. Mit Mn-o-Tolidin keine Reaktion, mit Indigo ein Oxydationswert, entsprechend 5 bis $10 \gamma \mathrm{O}_{3} / 1$.

fast negative Mn-o-Tolidin-Reaktion aufwies, während mit Indigo das Oxydationsvermögen der Lösung eindeutig messbar war.

Alle vorstehenden Beobachtungen deuten darauf hin, dass die Reaktionsverhältnisse bei der Ozonisierung von natürlichen Wässern - soweit wir sie mit den bisherigen analytischen Methoden erfassen können - noch 
nicht klar zu überblicken sind. Für die Praxis geht jedoch aus ihnen hervor, dass eine hohe Ozonzehrung eines Wassers, die mit der Mn-o-TolidinMethode festgestellt wird, noch keineswegs die Ozonisierung als Entkeimungsmethode a priori ausschliesst. Allerdings wird man als Massstab für die Bakterizidie des behandelten Wassers nicht mehr. die Mn-oTolidin-Methode verwenden können, sondern die Überwachung der Anlage zum Beispiel mit der Indigo-Methode vornehmen müssen. Damit dehnt sich der Anwendungsbereich der Ozonisierung gegenüber früheren Auffassungen erheblich aus. Trotzdem lassen die beträchtlichen Betriebskosten (vgl. SCHELler und GubELMANN [II]; SCHELler [22]) und die doch ziemlich komplizierte Apparatur das Verfahren auch in Zukunft wohl nur für mittlere und grössere zentrale Wasserversorgungen als geeignet erscheinen.

Soweit die Erfahrungen bisher reichen, ist das Ozonverfahren vor allem auch dort sehr vorteilhaft, wo ein Wasser durch Verunreinigungsstoffe geruchlich oder geschmacklich beeinträchtigt ist, da die starke Oxydationswirkung des Desinfektionsmittels die Geruchsträger meistens zerstört.

\section{Schlussfolgerungen}

Ein Desinfektionsverfahren für Wasser hat zwei gleichberechtigte Anforderungen zu erfüllen:

I. Die pathogenen Organismen im Wasser müssen abgetötet werden.

2. Das behandelte Wasser darf weder geruchlich noch geschmacklich verändert werden. Von dieser Forderung sollte man nur in Notfällen abweichen.

Feder Entkeimungsmethode für $W$ asser ist mit ibren speziffschen Eigenschaften ein bestimmter Anwendungsbereich zugewiesen, der durch den Wassercbemismus und durch die örtlichen Verbältnisse (Art des Netzaufbaues, $W$ artungsmöglichkeiten usw.) umgrenzt ist. Als Basis für die praktische Anwendung eines Verfahrens müssen folgende Grundlagen bekannt sein :

I. die Dosis-Wirkungs-Kurve oder Konzentrations-Wirkungs-Kurve des Desinfiziens für die in Betracht kommenden pathogenen Organismen;

2. die Abhängigkeit der Abtötungsgeschwindigkeit der pathogenen Organismen von den wichtigsten Aussenfaktoren, wie Temperatur, $\mathrm{pH}$, Mineralgehalt des Wassers;

3. die möglichen Einwirkungen der Inhaltsstoffe des behandelten Wassers auf das desinfizierende Agens. 
Die Einsatzgebiete der drei besprochenen Desinfektionsverfahren können zusammenfassend wie folgt umschrieben werden:

I. UV-Verfabren. Wasser dauernd klar. UV-Absorption im Wellenlängengebiet von $2600 \AA$ gering (arm an Huminstoffen und Eisen). Vor allem für Netze ohne Kontaktbehälter geeignet. Geräte direkt in Druckleitungen einschaltbar. Wartung gering und Funktionskontrolle einfach. Betriebsaufwand im Mittel um 5 Wh $/ \mathrm{m}^{3}{ }^{3}$ Keine Geruchs- oder Geschmacksbeeinträchtigung möglich. Nachwirkung des Desinfektionsmittels ausgeschlossen.

2. Silberperfabren. Keine speziellen Anforderungen an die Wasserqualität; sehr hohe Chloridgehalte (zum Beispiel über $50 \mathrm{mg} / \mathrm{l}$ ), Ca-Gehalte (z.B. über 40 französische Härtegrade) und tiefe $\mathrm{pH}$-Werte (unter 7) sind aber bei der Dimensionierung speziell zu berücksichtigen. Kontaktbehälter im Netz unentbehrlich. Grösse der Kontaktbehälter und Betriebsaufwand (Silberverbrauch) sind direkt voneinander abhängig. Bei durchschnittlichen Verhältnissen kann mit einem Betriebsaufwand von o,os g Silber $/ \mathrm{m}^{3}$ bei einer Kontaktzeit von I-2 Stunden gerechnet werden. Wartung der Silberdosierungsgeräte gering und Funktionskontrolle einfach. Geruchsund Geschmacksbeeinträchtigung nicht möglich. Lange Nachwirkung des Desinfektionsmittels im Wasser.

3. Ozonperfabren. Wasser mit geringem Gehalt an organischen Stoffen erwünscht. Hohe $\mathrm{pH}$-Werte (über 8) ungünstig. Möglichst kleiner Gehalt an Schwermetallen. Notwendige Kontaktzeit wenige Minuten. Betriebsaufwand (Ozonherstellung und Ozonlösung) um 60-70 W/g $\mathrm{O}_{3}$. Notwendige Einrichtungen verhältnismässig kompliziert. Wartung anspruchsvoller als bei den beiden vorgenannten Verfahren. Geruchs- und Geschmacksbeeinträchtigungen praktisch ausgeschlossen. Keine Nachwirkung des Desinfektionsmittels im Wasser.

\section{RÉSUMÉ}

Les procédés de désinfection de l'eau doivent remplir deux conditions également impératives:

$\mathrm{I}^{\circ}$ Les organismes pathogènes contenus dans l'eau doivent être détruits.

$2^{\circ}$ L'eau traitée ne doit présenter ni altération de goût ni d'odeur; en cas d'urgence seulement, on devrait déroger à ce principe.

Cbaque métbode de désinfection de l'eau, présentant des caractéristiques qui lui sont propres, a son champ d'application spécifique qui est déterminé par le chimisme de l'eau et les conditions 
locales (réseau de distribution, possibilités d'entretien, etc.). Il faut connaître les facteurs suivants pour décider de l'application d'un procédé déterminé:

$I^{\circ}$ Courbe dose/effet ou concentration/effet du désinfectant pour les organismes pathogènes devant être détruits.

$2^{\circ}$ Vitesse de destruction des organismes pathogènes en fonction des facteurs extérieurs les plus importants (température, $\mathrm{pH}$, teneur en substances minérales de l'eau).

$3^{\circ}$ Réactions possibles du désinfectant avec les constituants de l'eau traitée.

Les champs d'application des trois procédés de désinfection présentés peuvent être délimités d'une manière succincte comme suit:

I Procédé aux rayons ultraviolets. Eau claire en permanence. Faible absorption des rayons ultraviolets dans le domaine des longueurs d'onde de $2600 \AA$. Convient surtout pour réseaux sans bassins de contact. Incorporer les appareils directement dans les conduites sous pression. Entretien minime et contrôle du fonctionnement simple. Consommation moyenne environ $5 \mathrm{Wh} / \mathrm{m}^{3}$. Altération exclue du goût et de l'odeur de l'eau. Toute réaction secondaire due au désinfectant est exclue.

$2^{\circ}$ Procédé aux ions d'argent. L'eau ne doit présenter aucune qualité spéciale; toutefois, des teneurs très élevées en chlorures (par exemple supérieures à $50 \mathrm{mg} / \mathrm{l}$ ), en calcium (par exemple une dureté supérieure à 40 degrés français) et un $\mathrm{pH}$ trop bas (en-dessous de 7) augmentent le temps de contact. Bassins de contact indispensables dans le réseau. Les frais d'exploitation (consommation d'argent) sont corrélés aux dimensions des bassins de contact. Dans des conditions qualifiables de moyennes, il faut compter avec une consommation d'argent de $0,05 \mathrm{~g}$ d'argent $/ \mathrm{m}^{3}$ pour un temps de contact de $\mathrm{I}$ à 2 heures. Entretien minime et contrôle du fonctionnement facile des appareils de dosage de l'argent. Altération exclue de l'odeur et du goût de l'eau. Effet désinfectant de longue durée dans l'eau.

$3^{\circ}$ Procédé à l'ozone. De préférence pour eaux à faible teneur en substances organiques. pH élevé (au-dessus de 8) défavorable. Teneur en métaux lourds aussi faible que possible. Quelques minutes de contact seulement. Frais d'exploitation (fabrication et dissolution de l'ozone) équivalent à quelques 60 à $70 \mathrm{~W} / \mathrm{g}$ d'ozone. Appareillage relativement compliqué; entretien plus exigeant que pour les deux autres procédés précités. Altération du goût et de l'odeur de l'eau pratiquement exclue. Aucune réaction secondaire due au désinfectant.

\section{LITERATURVERZEICHNIS}

[I] Alder, M. G., und G. R. Hill, J. Amer. chem. Soc. 72, I884 (1950).

[2] ALHA, A. R., Acta path. microbiol. scand., Suppl. 65 (1946).

[3] Braidech, M. M., und F. H. Emery, J. Amer. Wat. Wks. Ass. 27, 557 (I935).

[4] Buchbinder, L., M. Soloway und E. B. Phelps, J. Bact. 42, 353 (I94I).

[s] Buchrolz, J., und A. v. JenNey, Zbl. Bakt. [I] 133, 299 (1934/35).

[6] Buffle, J. PH., Tech. sanit. municip. 45, 74 (1950). 
[7] Dorta-Schäppi, Y., und W. D. Treadwell, Helv. chim. Acta 32, 356 (r942).

[8] Duggard, B. M., und A. Hollaender, J. Bact. 27, 219 (I934).

[9] EHRismanN, O., und W. Nöthlin, Z. Hyg. Inf.-Krankh. 113, 597 (1932).

[io] GaUze, G. F., C. r. Acad. Sci. USSR 27, 722 (I940); cf. Brit. Abstr. [A] 3, 230 (1946).

[i i] Gubelmann, H., und H. Scheller, Monatsbull. schweiz. Ver. Gas- u. Wasserfachm. 33, 53 (I953).

[I2] HaAs, E., Biochem. Z. 282, 224 (I935).

[r3] HOlluta, J., und U. UNGER, Vom Wasser 21, I29 (1954).

[i4] Kelner, A., Proc. nat. Acad. Sci., Wash. 35, 73 (I949).

[is] Kelner, A., J. cell. comp. Physiol. 39, Suppl. I, i35 (I952).

[i6] Leiguarda, R. H., O. A. Peso und A. Z. R. De Palazzolo, Rev. Obr. sanit. Nac., B. Aires 13, 33 (I949); cf. Bull. Hyg., Lond. 25, I279 (I950).

[17] LuCKIEsh, M., Applications of Germicidal, Erythemal and Infrared Energy (v. Norstrand Comp., New York 1946).

[18] MCCULLOCH, E. C., Desinfection and Sterilization (Lea \& Febiger, Philadelphia 1945).

[i9] Newton, W. L., und M. F. Jones, J. Amer. Wat. Wks. Ass, 41, IO27 (I949).

[20] NÄGELI, C. v., N. Denkschr. schweiz. Ges. Naturw. 33, I (1893).

[2I] OHlmüller, R., Arb. Kais. Ges. Amt 8, 229 (I892).

[22] Scheller, H., Monatsbull. schweiz. Ver. Gas- u. Wasserfachm. 36, 73 (1956).

[23] Stumm, W., Helv. chim. Acta 38, 773 (1954).

[24] STUMm, W., Schweiz. Z. Hydrol. 18, 201 (1956).

[25] SüPfle, K., Zbl. Hyg. 1, I29 (1922).

[26] Swart-Füchtbauer, H., und A. Rippel-Baldes, Arch. Mikrobiol. 16, 358 (I95I).

[27] Toмcsik, J., Proc. Soc. exp. Biol., N. Y. 89, 459 (1955).

[28] Tor ricelli, A., Mitt. Lebensm. u. Hyg., Bern 38, 176 (1947).

[29] WeIss, J., Trans. Faraday Soc. 31, 668 (1935).

[30] Wuhrmann, K., und J. Meyrath, Schweiz. Z. Path. 18, 1060 (1955).

[31] Zehender, F, und W. Stumm, Mitt. Lebensm. u. Hyg., Bern 44, 206 (1953).

[32] ZimmermanN, W., Z. Hyg. 135, 403 (1952). 